\title{
Factores psicosociales que influyen en el proceso de compra al ser atendidos por personas con discapacidad cognitiva
}

León Felipe Collazos Salazar Universidad Autónoma de Occidente, UAO (Colombia) Ifcollazos@uao.edu.co

Sebastián Beltrán Díaz Universidad Autónoma de Occidente, UAO (Colombia) sebastian.beltran@uao.edu.co

Nyllireth Orjuela Rodríguez Universidad Autónoma de Occidente, UAO (Colombia) nyllireth.orjuela@uao.edu.co

\begin{abstract}
La investigación científica plantea un estudio cualitativo de las actitudes y los sentimientos que tienen los consumidores cuando son atendidos por personas en condición de discapacidad cognitiva y propone el análisis de la percepción que tienen los clientes respecto a las empresas que aplican esta práctica.

A partir de la revisión de estudios, se encuentra que para la ciudad de Cali en el año 2017 el porcentaje aproximado de personas con discapacidad cognitiva fue de un $2 \%$, equivalente a 5.082 personas. Además, los focos de investigación se centran únicamente en el impacto administrativo que tiene en las empresas la contratación de personas en condición de discapacidad

cognitiva, enfocándose en temas referentes a la inclusión social, la sostenibilidad y la responsabilidad social empresarial.
\end{abstract}




\section{Las nuevas narrativas, en el entorno social Universidad de La Laguna, diciembre de 2019}

Los resultados del proyecto, permiten identificar nuevas perspectivas. Primero, las actitudes y los sentimientos que tienen las personas ante este contexto, la percepción de los atributos de servicio que se ven afectados al momento de recibir el servicio y los factores psicosociales que influyen en el comportamiento del consumidor, como son la motivación y el aprendizaje. El análisis provee importantes datos para las empresas que contratan personas en esta condición, así como la incidencia en la imagen de la compañía y la alta probabilidad de recompra.

Finalmente, los hallazgos también pueden resultar de interés para otros grupos académicos, estudiantes de sociología, psicología, mercadeo y demás ramas del estudio del comportamiento humano, ya que permite entender de qué manera incide realmente este contexto de servicio sobre las percepciones de los consumidores, permitiendo generar nuevos prospectos investigativos que vislumbren nuevos resultados.

Keywords: Discapacidad Cognitiva; Factores Psicosociales; Percepción; Emociones; Sentimientos; Motivación; Aprendizaje.

\section{Introducción}

El propósito de la investigación es la identificación de los factores sociales y psicológicos que pueden llegar a influir en el comportamiento de compra del consumidor cuando está expuesto a un ambiente de servicio con personas en condición de discapacidad cognitiva. De igual manera, conocer la incidencia que tiene esta situación en la percepción de la empresa o el empleado.

La obtención de la información se logra mediante herramientas de recolección de datos cualitativas como Focus Group, Técnicas Proyectivas, Entrevistas en Profundidad y finalmente la Netnografía, específicamente con la aplicación de un Sentiment Text Analysis. 


\section{Las nuevas narrativas, en el entorno social \\ Universidad de La Laguna, diciembre de 2019}

Por otro lado, se ofrece una contextualización del problema en cuestión, brindando un acercamiento a la caracterización de esta población con discapacidad cognitiva en la ciudad de Cali, lo cual permite plantear la metodología de la investigación, para dar respuesta efectiva a los objetivos de la investigación científica.

\section{Problema de investigación}

A partir de la recolección de datos, se encuentran dos enfoques de estudio, por una parte, aquel que se fundamenta en la importancia de desarrollar estrategias de mercadotecnia para personas con discapacidad, y en otro, las ventajas que tiene para la empresas desde el punto de vista económico/financiero la inclusión de personas con esta condición en el ámbito laboral. La revista Portafolio (2015), en su artículo "Beneficios al contratar personas con Discapacidad", menciona cada beneficio y en qué leyes se fundamentan.

Por otro lado, teniendo en cuenta la información que se logró obtener mediante una investigación exploratoria en páginas web de entidades como el DANE (2017) (Departamento Administrativo Nacional de Estadística), en donde se evidencia una falencia en la caracterización de personas en condición de discapacidad y específicamente en la cognitiva, debido a que las condiciones de vida de algunas comunas es superior, referente a nivel socioeconómico y estilo de vida, asumiendo como hecho algunos aspectos sociales desfavorables, lo cual, en este caso, es un factor de error y por este motivo se habla de aproximación porcentual.

A través de esta falencia se logra identificar un porcentaje aproximado del número de ciudadanos en esta condición especial para la ciudad de Cali en el 2017, cercana a los 5.082 habitantes, lo cual equivale aproximadamente al $2 \%$ de la población. 


\section{Las nuevas narrativas, en el entorno social \\ Universidad de La Laguna, diciembre de 2019}

El anterior planteamiento revela una escasez en la información respecto a la caracterización de la población en condición de discapacidad cognitiva. Debido, a que se encuentra tan sólo evidencia de esta población en una caracterización realizada por la Alcaldía.

En este orden de ideas, estos hallazgos, permitieron conocer la falta de interés de entidades y también de la población en general para la identificación de los factores psicológicos y sociales que influyen en el comportamiento del consumidor cuando está expuesto a un contexto de servicio por parte de personas en condición de discapacidad cognitiva.

Teniendo en cuenta esta situación, se plantea el problema de investigación y es ¿De qué manera inciden los factores sociales y psicológicos, en la actitud del consumidor cuando es atendido por personas en condición de discapacidad cognitiva, y si este impacto está relacionado únicamente hacia la persona en condición de discapacidad cognitiva o tiene alguna repercusión en la percepción de la empresa en general?

Finalmente, los hallazgos de la investigación resultan de gran interés para empresas que tengan como iniciativa la contratación de personas con esta condición especial en su nómina, también para académicos, estudiantes de sociología, psicología, mercadeo y por último, la posibilidad de nuevos prospectos investigativos enfocados al estudio del comportamiento desde diferentes perspectivas, que se generen a partir de los resultados de la investigación científica.

\section{Objetivos}

\section{Objetivo general}

Conocer el impacto que tienen los factores sociales y psicológicos en el comportamiento de compra del consumidor cuando es atendido por empleados en condición de discapacidad cognitiva.

\section{Objetivos específicos}




\section{Las nuevas narrativas, en el entorno social \\ Universidad de La Laguna, diciembre de 2019}

- Conocer las actitudes y los sentimientos de los consumidores y la incidencia en la percepción de la marca referente al servicio al cliente, al momento de exponerse a un ambiente de servicio con personas en condición de discapacidad cognitiva.

- Indagar a cerca de la percepción del consumidor respecto a una empresa que tiene vinculada a un empleado en condición de discapacidad cognitiva.

- Identificar qué factores psicosociales influyen en el proceso de compra de una persona cuando es atendida por un empleado en condición de discapacidad cognitiva.

\section{Marco teórico}

\subsection{Comportamiento del consumidor.}

Kotler (2003) muestra la definición del concepto de comportamiento del consumidor como: " el estudio o análisis de la forma en que las personas, los grupos y las empresas seleccionan, adquieren, utilizan y disponen de bienes, servicios, ideas o experiencias para satisfacer sus necesidades y deseos" (pág.6)

El autor también sostiene que, primero que todo se presenta la intención de compra, la cual puede variar por aspectos sociales como la presión social, es decir, si una persona considera que la decisión que se debe tomar debería ser otra a la que el consumidor considera, podría influir en el comportamiento de la persona que realizará la compra. Otro aspecto que interfiere en este punto, son los factores inesperados, como puede ser cambios de los precios de los productos. Finalmente, cuando el consumidor ha estado expuesto a estos factores, viene la decisión de compra, que ya es algo relativo.

En este sentido, la decisión de compra del consumidor que se encuentra expuesto a un contexto de servicio con personas en condición de discapacidad 


\section{Las nuevas narrativas, en el entorno social Universidad de La Laguna, diciembre de 2019}

cognitiva, se puede observar y comparar con la de otros consumidores, para así ener idea de los diversos comportamientos que se presentan.

\subsection{Percepción de marca}

Según Keller (2008), el valor capital de la marca basado en el cliente se puede definir con los sentimientos que evoca una marca en la persona. Dice que:

Los sentimientos hacia la marca son las respuestas y reacciones emocionales de los clientes, y se relacionan con el valor social que la marca evoca en ellos. ¿Qué sentimientos son concitados por el programa de marketing para la marca o por otros medios? ¿Cómo afecta la marca la sensibilidad de los clientes acerca de sí mismos y de sus relaciones con los demás? Estos sentimientos pueden ser leves o intensos, positivos o negativos. (p.68)

Según lo anterior, se evidencia que los sentimientos hacia una marca pueden efectivamente definir el concepto o percepción que se tiene de ella, el autor también menciona que existen dos tipos de emociones vinculadas a la percepción y sentimientos hacia una marca, "La aprobación social" y "El Autorrespeto", estos darían respuesta a la percepción del cliente.

\section{Estado del arte}

Como se mencionó en el desarrollo del problema y la justificación, el estado del arte del tema que se está tratando en la presente investigación es escaso. Existen algunas perspectivas que se han abordado respecto a las personas con discapacidad cognitiva y el mundo laboral, hasta el momento no se han tocado temas más específicos como el comportamiento del consumidor y la percepción de la marca referente al mismo tema.

De igual manera, algunos de los estudios previos que se han realizado, están enfocados por ejemplo a las habilidades que debe tener una persona en condición de discapacidad cognitiva para poder empezar una vida laboral, en este caso los autores Vilà Suñé, Pallisera, Fullana Noell (2012): 


\section{Las nuevas narrativas, en el entorno social Universidad de La Laguna, diciembre de 2019}

Desde una perspectiva general, la inclusión laboral de personas con discapacidad depende en gran medida del análisis por parte del contratante en relación a los procesos educativos que las personas con esta condición hayan tenido, principalmente en su preparación secundaria, debido a que esto es determinante para las oportunidades que se les puedan presentar posteriormente en el mercado laboral e incluso para el desarrollo, la construcción y la autonomía personal. (p.87)

Otro aspecto importante para determinar una inclusión laboral de personas con estas condiciones, según estos autores Vilà Suñé, Pallisera, Fullana Noell (2012), consiste en que:

Varios estudios centrados en los factores facilitadores de la inclusión laboral de las personas con discapacidad intelectual inciden en subrayar el papel que tienen las habilidades socio personales (toma de decisiones, autoestima, responsabilidad, resolución de problemas) en el éxito de estos procesos (Alomar, 2004; Rius, 2005; Vilà y Pallisera, 2006; Vilà et al, 2007). Estas habilidades forman parte de los componentes que se consideran claves en la autodeterminación: toma de decisiones, resolución de problemas, habilidades para establecer y conseguir objetivos, habilidades de independencia, asumir riesgos y seguridad, habilidades de autoobservación, evaluación y refuerzo, habilidades de autoinstrucción, habilidades de autodefensa y liderato, locus de control interno, atribuciones positivas de eficacia y de expectativas de resultados, autoconciencia y autoconocimiento (Wehmeyer, 1998). La investigación muestra que un mayor dominio de estas habilidades está relacionado con mejores posibilidades de inclusión sociolaboral en los procesos de tránsito a la vida adulta de las personas con discapacidad intelectual (Wehmeyer y Palmer, 2003; Test et al, 2009; Madaus, Gerber y Price, 2008).

Otra de las perspectivas que se mencionan en los estudios históricos, hace énfasis en cómo llegar a al mercado de personas con discapacidad cognitiva y qué estrategias aplicar a este target. Entonces, la inclusión laboral con personas en situación de discapacidad cognitiva puede representar para la sociedad un reto de adaptación al comienzo de vida profesional. Para que esto se pueda dar de manera eficaz, es necesario desarrollar un plan de mercadeo social. Kotler 


\title{
Las nuevas narrativas, en el entorno social \\ Universidad de La Laguna, diciembre de 2019
}

(2008) desarrolló una estructura de plan, donde menciona los pasos que se deben tener en cuenta para lograrlo.

\begin{abstract}
En primera instancia es importante tener un propósito y un enfoque, es decir, qué impacto social se desea, en cuál población y con qué solución, además de esto, es necesario evaluar el mercado y la situación donde se encuentra. Luego se debe tener claro el target con el que se quiere trabajar. En este caso, son las personas en condiciones de discapacidad cognitiva. Se debe caracterizar el mercado deseado y postular los objetivos que se desean alcanzar. Luego, se planifican estratégicamente acciones de la mezcla de mercado y finalmente se debe proponer un presupuesto previsto para la puesta en marcha de dichas estrategias. (cáp.2)
\end{abstract}

\section{Metodología}

En la investigación científica se presentan hallazgos cualitativos que se lograron obtener a través de técnicas utilizadas principalmente para conseguir información que es de gran interés para los investigadores. Dicha información es relevante para determinar posteriormente las hipótesis planteadas dentro del proyecto.

Dentro de la investigación cualitativa, como se acaba de mencionar, existen una serie de herramientas que permiten la identificación de hallazgos importantes al momento de realizar la investigación, Malhotra (2004) sostiene que dentro de la investigación de mercados exploratoria, se encuentran herramientas para su correcto desarrollo, tales como:

- Focus Group: consiste en una entrevista, de forma no estructurada y natural, que un moderador capacitado realiza a un pequeño grupo de participantes. El moderador guía el debate. El principal objetivo de los Focus Group consiste en obtener información al escuchar las opiniones de un grupo de personas del mercado meta sobre temas de interés para 


\section{Las nuevas narrativas, en el entorno social \\ Universidad de La Laguna, diciembre de 2019}

el investigador. El valor de la técnica reside en los hallazgos inesperados que a menudo se obtienen de un debate grupal que fluye libremente.

Las sesiones de grupo se dividen en 7 Focus Group de 5 personas cada uno, con un segmento de mercado de personas que habiten en la ciudad de Santiago de Cali, con edades que oscilan entre los 18 a 56 años y quienes no han tenido una experiencia de compra con personas en condición de discapacidad cognitiva.

Debido a la posible presión social durante el desarrollo de las sesiones de grupo, se considera pertinente para la investigación, incluir también el uso de las entrevistas en profundidad, las cuales permiten indagar y recolectar información para la investigación, reforzando y añadiendo valiosa información a lo ya hallado durante los Focus Group.

- Entrevistas a profundidad: Este tipo de técnica no estructurada, así como lo menciona Malhotra (2008) se aplicará con "la finalidad de indagar las motivaciones, creencias, actitudes y sentimientos subyacentes acerca de un tema" (p.158).

Para esta investigación es pertinente usar dicha técnica, dado que se busca conocer factores sociales y psicológicos que inciden en los consumidores cuando se exponen a un contexto de servicio con personas en condición de discapacidad cognitiva, para así determinar de qué manera influye en sus actitudes y comportamientos y qué acciones realizan después de ser atendidos por un empleado en dicha condición.

La entrevista en profundidad resulta relevante para el proyecto, debido al contexto social que involucra la investigación científica, dado que las personas entrevistadas se pueden cohibir de dar opiniones al estar dentro de una sesión de grupo. Por otro lado, al estar en una entrevista de manera personal, se puede obtener mayor respuesta, sin que exista presión social. 


\section{Las nuevas narrativas, en el entorno social \\ Universidad de La Laguna, diciembre de 2019}

Para el desarrollo de la investigación, se realizaron 15 entrevistas en profundidad a personas que de igual manera no han sido atendidas por personas en condición de discapacidad cognitiva, pues de esta manera se sigue el hilo conductor propuesto con los Focus Group.

De igual modo, para lograr una mejor comprensión y facilitar el discurso no apegado al estereotipo sobre temas que generan tabúes sociales, así como favorecer la expresión de ideas y emociones difíciles de expresar a través del lenguaje verbal, se hace pertinente el uso de las técnicas proyectivas para complementar la información encontrada en los Focus Group.

Técnicas proyectivas: Malhotra (2008) afirma que este tipo de técnica "anima a los participantes a proyectar sus motivaciones, creencias, actitudes o sentimientos subyacentes con respecto a los temas de interés" (p.163) .

Dentro de la investigación científica, resulta pertinente este tipo de técnica porque favorece la expresión de ideas y sentimientos, facilitando la proyección de manera indirecta de las propias motivaciones, actitudes o sentimientos que se tengan cuando el consumidor recibe el servicio por parte de una persona en condición de discapacidad cognitiva.

Para lo cual, se plantea la realización de una técnica proyectiva de asociación por imagen, donde se presenta a los participantes diferentes imágenes de empleados en condición de discapacidad cognitiva estando en un contexto de servicio. Logrando que los integrantes del Focus Group y directamente de las técnicas proyectivas, den respuesta a los objetivos que se desean alcanzar en el proyecto.

La población objetivo a la cual está dirigida esta técnica, se centra en personas de la ciudad de Santiago de Cali, en edades entre 18 a 56 años, que pertenezcan a los niveles socioeconómicos 3, 4, 5 y 6 . 


\section{Las nuevas narrativas, en el entorno social Universidad de La Laguna, diciembre de 2019}

En este caso, se utilizaron las anteriores técnicas, las cuales se llevaron a cabo de una manera exitosa y permitieron cumplir a cabalidad la obtención de una respuesta a los objetivos planteados. Además de los Focus Group, las Técnicas proyectivas y las Entrevistas a Profundidad, se quiso utilizar una técnica adicional para contrastar los resultados con los anteriormente obtenidos, esta técnica se trata de:

- Netnografía (Sentiment Text Analysis): Esta herramienta de investigación de mercados, según Hutto y Gilbert (2016) (P. 217), consiste en enfoques de análisis de sentimientos que dependen en gran medida de un léxico subyacente que se orienta semánticamente como positivo o negativo. Esto se realiza a través de una investigación lingüística y conteo de palabras, principalmente mediante las redes sociales.

En este caso específico, se utilizó la red social Facebook, mediante una imagen donde se podía observar a una mesera con Síndrome de Down atendiendo en un restaurante llamado D'Cafe. La imagen contiene más de 300 comentarios, de los cuales se seleccionaron los que estaban relacionados directamente con el contexto de servicio con personas en condición de discapacidad cognitiva. Esta herramienta fue de gran utilidad para vislumbrar las percepciones y conocer los sentimientos de las personas mediante una red social.

Ahora, posterior a los hallazgos de esta investigación, se puede determinar un campo más amplio de análisis y estudios referentes al tema de personas con discapacidad cognitiva, debido a que se evidencia el claro cumplimiento de los objetivos propuestos y permite generar a diferentes grupos de interés hipótesis y estudios adicionales y nuevas perspectivas investigativas.

A continuación se presentan los resultados cualitativos.

\section{Resultados y prospectiva}

\section{ANÁLISIS OBJETIVO 1}




\section{Las nuevas narrativas, en el entorno social \\ Universidad de La Laguna, diciembre de 2019}

- Conocer las actitudes y los sentimientos de los consumidores y la incidencia en la percepción de la marca referente al servicio al cliente, al momento de exponerse a un ambiente de servicio con personas en situación de discapacidad cognitiva.

Las personas, en su mayoría, sentirían alegría y tendrían una actitud de amabilidad y respeto al ver la inclusión que las empresas están generando cuando emplean personas en condición de discapacidad cognitiva. El hecho de inclusión, las ganas de superación de estas personas y la comprensión que tendrían, son factores que los haría sentir cómodos como clientes en este contexto.

Otro de los sentimientos encontrados fue admiración (gran mayoría) y solidaridad; la indiferencia fue casi irrelevante en este sentido. Los sentimientos negativos no formaron parte de los hallazgos encontrados en el análisis de los Focus Group ni la Entrevista en Profundidad.

En cuanto a la incidencia en la percepción referente al servicio prestado por parte de estos empleados, los atributos que podrían verse afectados negativamente son: el tiempo de espera, al incrementar en cierta medida y la calidad del servicio referente al contacto directo con el empleado en esta condición debido a la incertidumbre por parte del cliente ante los sentimientos del empleado, pues consideran que los harían sentir incómodos, y este hecho haría que ellos mismos se sientan incómodos.

Por otro lado, la calidad del servicio y la amabilidad, son atributos que, a pesar de que para la mayoría de personas mejorarían en una empresa donde haya un empleado con discapacidad cognitiva, ya que los clientes consideran que los empleados en dicha condición son más amables y pacientes, se hallaron algunos comentarios donde se exponían posibles problemas de comunicación, lo cual perjudica la calidad del servicio y el cliente o cliente potencial quedaría expuesto a una situación donde no sabrían cómo actuar.

\section{ANÁLISIS OBJETIVO 2}


- Indagar a cerca de la percepción del consumidor respecto a una empresa que tiene vinculada a un empleado en condición de discapacidad cognitiva.

La percepción que tuvieron todos los participantes de los Focus Group y las Entrevistas en Profundidad, respecto a las empresas que contratan personas con discapacidad cognitiva, fue positiva, a tal punto que en la totalidad de los comentarios se afirmó que estas empresas fomentan la inclusión y que son empresas las cuales tienen en los valores y principios corporativos el factor de inclusión.

Es importante mencionar que el hecho de que empleen a estas personas, hace posible que los clientes vuelvan a comprar a estos establecimientos, para así apoyar las oportunidades que brindan a las personas en condición de discapacidad cognitiva.

\section{ANÁLISIS OBJETIVO 3}

- Identificar qué factores psicosociales influyen en el proceso de compra de una persona cuando es atendida por un empleado en condición de discapacidad cognitiva.

Dentro de los factores psicológicos que influyen en la decisión de compra de una persona cuando es atendida por un empleado en condición de discapacidad cognitiva, se encuentra la motivación, debido a que existe un grupo de personas que consideran que el hecho de que hayan empleados con discapacidad cognitiva, los motiva a ir a ese sitio e incluso les haría comprar ahí únicamente por el hecho de que haya un empleado con dicha condición.

Otro factor psicológico que se encontró como influyente en el proceso de compra, es la percepción. Varias personas estuvieron de acuerdo en que una empresa que contrate a personas en condición de discapacidad cognitiva y esté implementando la inclusión social, es una empresa que vale la pena visitar y cambiaría su perspectiva e incluso haría que visitaran el lugar. Por tal motivo, al 


\section{Las nuevas narrativas, en el entorno social \\ Universidad de La Laguna, diciembre de 2019}

tener este cambio en la mente del cliente potencial, está de alguna manera influyendo en la recordación del cliente y haciendo que perciba a la organización de manera positiva.

Todo esto tiene en la mente del consumidor un factor psicológico clave, que es el aprendizaje. Luego de que el cliente percibe diferente a una empresa, se encuentra motivado a adquirir un producto/servicio, ha existido un cambio mental y ese cliente va a incurrir a una recompra, eso quiere decir que existió un proceso de aprendizaje en el contexto que se encontraba.

Finalmente esto hace que el cliente tenga cambios en sus convicciones y actitudes, en este caso hacia una empresa que emplee personas con discapacidad cognitiva o bien sea hacia el empleado como tal.

Desde los factores sociales que influyen, se encontró que los sujetos que se mencionaron, todos llegan a influir de alguna u otra manera en las personas, unos más que otros, como son los padres y la pareja sentimental. De esta manera, se dice que socialmente hablando, los grupos de referencia y la familia, son sujetos con un grado de influencia alto en la decisión de compra de una persona, en este caso, para adquirir un producto o servicio en un establecimiento con personas en condición de discapacidad cognitiva.

\section{Netnografía (Sentiment Text Analysis).}

Se realizó una Netnografía, específicamente la herramienta de investigación cualitativa llamada "Sentiment Text Analysis". Esto consistió en la transcripción de 253 comentarios de una publicación de la red social Facebook, donde se muestra la atención de una mesera con Síndrome de Down. La publicación cuenta con 400 comentarios aproximadamente, de los cuales se tomaron únicamente los que se referían a la empleada de alguna manera, los que hablaban sobre la empresa o cualquier impresión referente a la imagen, los comentarios que no fueron tomados son los que únicamente etiquetaban a otras personas. 


\section{Las nuevas narrativas, en el entorno social \\ Universidad de La Laguna, diciembre de 2019}

Donde se logró observar que 199 personas tuvieron comentarios positivos respecto a la publicación, donde se evidenciaron palabras referentes a la empleada como: Hermosa, capaz, independiente, buena persona, atenta y demás frases que la identificaban como una persona que sale adelante y enorgullece a las personas.

Hubo 18 comentarios neutros, donde se clasificaron así los que no decían nada positivo ni negativo respecto a la publicación, por ejemplo comentarios como: "Les rebajan impuestos", "las empresas están en la obligación de hacerlo".

Finalmente, los comentarios negativos ascendieron a 36, superando así los neutros. Aquí se clasificaron principalmente comentarios irónicos como "servicio especial”, “¿cuál es la especialidad del día? También comentarios de burla hacia el servicio que presta la empleada.

De esta manera se logra concluir que en su mayoría, los comentarios positivos de las personas en general son más relevantes que los negativos y neutros permitiendo reforzar el análisis que se realizó en los Focus Group y Entrevistas en Profundidad, permitiendo estipular las respectivas hipótesis para análisis cuantitativo.

\section{Prospectiva}

Teniendo en cuenta la realidad de la generación de empleo en Colombia, es clave incorporar programas de capacitación para incrementar el personal en condición de discapacidad cognitiva, ya que como se muestra en la investigación, tiene una alta incidencia en la preferencia del consumidor hacia empresas que cuentan con este personal. Así, como el recibir un servicio por personas con dicha condición genera en su gran mayoría emociones y percepciones positivas, que están relacionadas directamente con el servicio ofrecido.

Por otra parte, extender estudios dedicados al análisis de otros factores que influyen dentro del comportamiento del consumidor, empleando herramientas de 


\section{Las nuevas narrativas, en el entorno social \\ Universidad de La Laguna, diciembre de 2019}

neuromarketing que puedan entregar información aún más detallada de la percepción del consumidor cuando recibe un servicio por parte de personas en condición de discapacidad cognitiva.

En síntesis, se deben tener en cuenta oportunidades de desarrollo para el personal en condición de discapacidad cognitiva en empleos que estén vinculados con la atención directa al cliente, siendo un factor clave de éxito para la percepción de la empresa y el servicio ofrecido.

\section{Referencias bibliográficas}

Portafolio. ( 13 de septiembre de 2015). "Beneficios al contratar personas con discapacidad". Recuperado de http://www.portafolio.co/opinion/redaccionportafolio/beneficios-contratar-personas-discapacidad-3733

Malhotra, N. Investigación de Mercados. Quinta Edición. México: Ed. Pearson - Prentice Hall. Retomado de http://www.cars59.com/wpcontent/uploads/2015/09/Investigacion-de-Mercados-Naresh-Malhotra.pdf. ISBN: 978-970-26-1185-1

Kotler, P., y Armstrong, G.(2003). Fundamentos de Marketing. $6^{\circ}$ Edición. México: Ed. Pearson Prentice Hall.

Vilá, M., Pallisera, M., Fullana, J. (2012). La inclusión laboral de los jóvenes con discapacidad intelectual: un reto para la orientación psicopedagógica. Revista Española de Orientación y Psicopedagogía.

HUTTO, C.J. GILBERT, Eric. VADER: A Parsimonious Rule-based Model for Sentiment Analysis of Social Media Text [En línea]. En: Eighth International Conference on Weblogs and Social Media. 20 de abril de 2016. P. 217. [Consultado el 25 de abril de 2018]. Disponible en internet: file://C:/Users/Sebasti\%C3\%A1n/Downloads/8109-37749-1-PB.pdf 